\title{
Ovoids of the Hermitian surface in odd characteristic
}

\author{
Luca Giuzzi and Gábor Korchmáros* \\ Dedicated to Professor Adriano Barlotti on the occasion of his 80 th birthday
}

\begin{abstract}
We construct a new ovoid of the polar space arising from the Hermitian surface of $\operatorname{PG}\left(3, q^{2}\right)$ with $q \geqslant 5$ odd. The automorphism group $\Gamma$ of such an ovoid has a normal cyclic subgroup $\Phi$ of order $\frac{1}{2}(q+1)$ such that $\Gamma / \Phi \cong \operatorname{PGL}(2, q)$. Furthermore, $\Gamma$ has three orbits on the ovoid, one of size $q+1$ and two of size $\frac{1}{2} q(q-1)(q+1)$.
\end{abstract}

Key words. Ovoid, Hermitian surface, polar space, automorphism group.

2000 Mathematics Subject Classification. Primary: 51E20, Secondary: 51A50

\section{Introduction}

The concept of ovoid and its generalisations have played an important role in finite geometry since the fifties. By a beautiful result of A. Barlotti [2] and G. Panella [10], every ovoid in $\operatorname{PG}(3, q)$ with $q$ odd is an elliptic quadric. This is a generalisation of Segre's famous theorem [11] stating that every oval in $\mathrm{PG}(2, q)$, with $q$ odd, is a conic. Ovoids of finite classical polar spaces have been intensively investigated, especially in the last two decades, see [1], [3], [4], [5], [9], [12], [13], [14] and the recent survey paper [15]. In this paper we are concerned with ovoids of the polar space determined by a non-degenerate Hermitian surface $\mathscr{H}\left(3, q^{2}\right)$ of $\mathrm{PG}\left(3, q^{2}\right)$.

An ovoid $\mathcal{O}$ of the polar space arising from $\mathscr{H}\left(3, q^{2}\right)$ is a set of $q^{3}+1$ points in $\mathscr{H}\left(3, q^{2}\right)$ which meets every generator (that is, every line contained in $\left.\mathscr{H}\left(3, q^{2}\right)\right)$ in exactly one point. The intersection of $\mathscr{H}\left(3, q^{2}\right)$ with any non-tangent plane provides an ovoid-namely, the classical ovoid of $\mathscr{H}\left(3, q^{2}\right)$. Existence of non-classical ovoids of $\mathscr{H}\left(3, q^{2}\right)$ was pointed out by Payne and Thas [16], who constructed a non-classical ovoid $\mathcal{O}^{\prime}$ from the classical one $\mathcal{O}$ by replacing the $q+1$ points of $\mathcal{O}$ lying in a chord $\ell$ by the common points of $\mathscr{H}\left(3, q^{2}\right)$ with the polar line $\ell^{\prime}$ of $\ell$. A straightforward generalisation of this procedure consists in replacing a number of chords of $\mathcal{O}$, each with its own polar line. The condition for the resulting set to be an ovoid is easily

* The present research was performed within the activity of G.N.S.A.G.A. of the Italian INDAM with the financial support of the Italian Ministry M.I.U.R., project "Strutture geometriche, combinatorica e loro applicazioni”, 2001-02. 
stated: the replaced chords must pairwise intersect outside of $\mathcal{O}$. The above procedure will be called derivation or multiple derivation according to one or more chords being replaced.

In this paper, we construct an ovoid $\mathcal{O}$ of $\mathscr{H}\left(3, q^{2}\right)$ for every odd $q \geqslant 5$ which cannot be obtained either by derivation or by multiple derivation. We also determine the automorphism group of $\mathcal{O}$, as given by the subgroup of $\operatorname{PGU}\left(4, q^{2}\right)$ preserving $\mathcal{O}$.

\section{Preliminary results on ovoids of the Hermitian surface}

Let $\mathscr{H}\left(3, q^{2}\right)$ be a non-degenerate Hermitian surface in $\operatorname{PG}\left(3, q^{2}\right)$. It is well known, see $\left[6\right.$, Chapter 19], that $\mathscr{H}\left(3, q^{2}\right)$ can be reduced by a non-singular linear transformation to the canonical form $X_{0}^{q} X_{3}+X_{0} X_{3}^{q}+u X_{1}^{q+1}+v X_{2}^{q+1}=0$, where $u, v \in \mathbb{F}_{q}$ are non-zero elements. The linear collineation group of $\mathrm{PG}\left(3, q^{2}\right)$ preserving $\mathscr{H}\left(3, q^{2}\right)$ is $\operatorname{PGU}\left(4, q^{2}\right)$. See [8] for a classification of the subgroups of $\operatorname{PGU}\left(4, q^{2}\right)$. We shall rely only upon an existence theorem for subgroups of homologies, as stated in the following lemma.

Lemma 2.1. Let $\alpha$ be a non-tangent plane to $\mathscr{H}\left(3, q^{2}\right)$ and $A$ its pole under the unitary polarity associated with $\mathscr{H}\left(3, q^{2}\right)$. Then the $(\alpha, A)$ homology group of $\operatorname{PGU}\left(3, q^{2}\right)$, that is, the maximal subgroup of $\mathrm{PGU}\left(3, q^{2}\right)$ consisting of homologies with axis $\alpha$ and centre $A$, is a cyclic group of order $q+1$.

We shall also need a characterisation of ovoids which can be obtained by multiple derivation.

Lemma 2.2. Let $\mathcal{O}^{\prime}$ be an ovoid of $\mathscr{H}\left(3, q^{2}\right)$. A necessary and sufficient condition for $\mathcal{O}^{\prime}$ to be obtainable from a classical ovoid $\mathcal{O}$ of $\mathscr{H}\left(3, q^{2}\right)$ through multiple derivation is that $\mathcal{O}^{\prime}$ is preserved by the $(\alpha, A)$ homology group of $\operatorname{PGU}\left(3, q^{2}\right)$ for a non-tangent plane $\alpha$ and its pole $A$.

Proof. Choose a pair $(\alpha, A)$ consisting of a non-tangent plane $\alpha$ to $\mathscr{H}\left(3, q^{2}\right)$ and the pole $A$ of $\alpha$ under the unitary polarity associated with $\mathscr{H}\left(3, q^{2}\right)$. Let $\mathcal{O}$ be the classical ovoid given by all common points of $\mathscr{H}\left(3, q^{2}\right)$ and $\alpha$. Denote by $\Psi$ the homology group of $\operatorname{PGU}\left(3, q^{2}\right)$ with axis $\alpha$ and centre $A$. It is easily verified that if an ovoid $\mathcal{O}^{\prime}$ arises from $\mathcal{O}$ by (multiple) derivation, then $\Psi$ preserves $\mathcal{O}^{\prime}$. Conversely, we prove that if $\Psi$ preserves an ovoid $\mathcal{O}^{\prime}$ different from $\mathcal{O}$, then $\mathcal{O}^{\prime}$ can be obtained from $\mathcal{O}$ by (multiple) derivation. Let $P \in \mathcal{O}^{\prime}$ be a point not on $\alpha$. Then the orbit of $P$ under $\Psi$ consists of the common points of $\mathscr{H}\left(3, q^{2}\right)$ and the line $\ell^{\prime}$ joining $A$ and $P$. Hence, $\mathscr{H}\left(3, q^{2}\right) \cap \ell^{\prime}$ is contained in $\mathcal{O}^{\prime}$. Let now $\ell_{1}^{\prime}, \ldots, \ell_{m}^{\prime}$ be the lines through $A$ which meet $\mathcal{O}^{\prime}$ outside $\alpha$, and let $\ell_{1}, \ldots, \ell_{m}$ be their corresponding polar lines. The latter lines are chords of the Hermitian curve $\mathscr{H}\left(2, q^{2}\right)=\mathcal{O}$, cut out on $\mathscr{H}\left(3, q^{2}\right)$ by the plane $\alpha$, and any two of them intersect outside $\mathscr{H}\left(2, q^{2}\right)$. This proves that $\mathcal{O}^{\prime}$ arises from $\mathcal{O}$ by multiple derivation. 


\section{The construction}

We assume $q \geqslant 5$ to be odd and write the equation of the Hermitian surface $\mathscr{H}\left(3, q^{2}\right)$ in its canonical form

$$
X_{3}^{q} X_{0}+X_{3} X_{0}^{q}+2 X_{2}^{q+1}-X_{1}^{q+1}=0 .
$$

The starting point of our construction is the following lemma.

Lemma 3.1. Let $(x, y)$ satisfy the relation

$$
y^{q}+y+x^{(q+1) / 2}=0 .
$$

Then the point $\left(1, x, y, y^{2}\right)$ lies on $\mathscr{H}\left(3, q^{2}\right)$.

Proof. If $(x, y)$ satisfies (3.2), then the polynomial identity

$$
\left(Y^{q}+Y-X^{(q+1) / 2}\right)\left(Y^{q}+Y+X^{(q+1) / 2}\right)=Y^{2 q}+2 Y^{q+1}+Y^{2}-X^{q+1}
$$

implies that $y^{2 q}+2 y^{q+1}+y^{2}-x^{q+1}=0$. The geometric interpretation of this equation is that the point $\left(1, x, y, y^{2}\right)$ lies on $\mathscr{H}\left(3, q^{2}\right)$.

Lemma 3.2. Let $x \in \mathbb{F}_{q^{2}}^{*}$. Then Equation (3.2) has either $q$ or 0 solutions in $y \in \mathbb{F}_{q^{2}}$, according as $x$ is a square or a non-square in $\mathbb{F}_{q^{2}}$.

Proof. We first prove that if $(x, y)$, with $x, y \in \mathbb{F}_{q^{2}}$, satisfies (3.2), then $x$ is the square of an element of $\mathbb{F}_{q^{2}}$. The assertion holds trivially for $x=0$; hence, we may assume that $x \neq 0$. Since $y^{q}+y \in \mathbb{F}_{q}$, we have $-x^{(q+1) / 2} \in \mathbb{F}_{q}$, whence $\left(x^{(q+1) / 2}\right)^{q-1}=1$. On the other hand, $x \neq 0$ is a square in $\mathbb{F}_{q^{2}}$ if and only if $x^{\left(q^{2}-1\right) / 2}=1$, which proves the assertion. Conversely, let $x$ be a square element of $\mathbb{F}_{q^{2}}$, and take $\xi \in \mathbb{F}_{q^{2}}$ such that $x=\xi^{2}$. By $[7,1.19]$, the equation $y^{q}+y=\xi^{q+1}$ has exactly $q$ solutions in $\mathbb{F}_{q^{2}}$. Hence, $y^{q}+y=x^{(q+1) / 2}$ holds for exactly $q$ values $y \in \mathbb{F}_{q^{2}}$. This completes the proof.

Let $\Sigma$ denote the set of all pairs $(x, y)$ with $x, y \in \mathbb{F}_{q^{2}}$ satisfying (3.2).

Lemma 3.3. The set $\Sigma$ has size $\frac{1}{2} q\left(q^{2}+1\right)$.

Proof. The number of squares in $\mathbb{F}_{q^{2}}$, zero included, is $\left(q^{2}+1\right) / 2$. Thus, the assertion follows from Lemma 3.2 together with a counting argument.

We embed $\Sigma$ in $\operatorname{PG}\left(3, q^{2}\right)$ by means of the map $\varphi:(1, x, y) \mapsto\left(1, x, y, y^{2}\right)$. Some properties of the embedded set are collected in the following two lemmas. 
Lemma 3.4. Let $\Delta$ be the set of all points $\left(1, x, y, y^{2}\right)$ of $\operatorname{PG}\left(3, q^{2}\right)$ with $(x, y) \in \Sigma$, together with the point $(0,0,0,1)$. Then

I) $\Delta$ has size $\frac{1}{2}\left(q^{3}+q+2\right)$;

II) The plane $\pi$ with equation $X_{1}=0$ intersects $\Delta$ in a set $\Delta_{1}$ of size $q+1$. The set $\Delta_{1}$ is the complete intersection in $\pi$ of the conic $\mathscr{C}$ with equation $X_{0} X_{3}-X_{2}^{2}=0$ and the Hermitian curve $\mathscr{H}\left(2, q^{2}\right)$ with equation $X_{0}^{q} X_{3}+X_{0} X_{3}^{q}+2 X_{2}^{q+1}=0$;

III) The Baer involution $\beta:=\left(X_{0}, X_{2}, X_{3}\right) \mapsto\left(X_{0}^{q},-X_{2}^{q}, X_{3}^{q}\right)$ of $\pi$ preserves both $\mathscr{C}$ and $\mathscr{H}\left(2, q^{2}\right)$. The associated Baer subplane $\pi_{0}$ of $\pi$ meets $\mathscr{H}\left(2, q^{2}\right)$ in $\Delta_{1}$;

IV) $\Delta_{1}$ lies in $\pi_{0}$ and consists of all the points of a conic $\mathscr{C}_{0}$ of $\pi_{0}$.

Proof. The lemma is a consequence of straightforward computations.

Lemma 3.5. The point $U=(0,1,0,0)$ is not in $\Delta$. Furthermore,

i) A line through $U$ meets $\Delta$ in either $\frac{1}{2}(q+1)$ or 1 or 0 points. More precisely, there are exactly $q^{2}-q$ lines through $U$ sharing $\frac{1}{2}(q+1)$ points with $\Delta$, and $q+1$ lines having just one point in $\Delta$. The former lines meet the plane $\pi$ in the points of the conic $\mathscr{C}$ not lying on $\Delta_{1}$; the latter in the points of $\Delta_{1}$;

ii) A plane through $U$ meets $\Delta$ in either $q+1$ or $\frac{1}{2}(q+1)$ or 0 points;

iii) A plane missing $U$ meets $\Delta$ in at most $q^{2}+1$ points.

Proof. In order to prove ii), take a point $P\left(1, x, y, y^{2}\right)$ in $\Delta$ and consider the line $\ell$ through $U$ and $P$. The point $P_{t}\left(1, x+t, y, y^{2}\right)$, for $t \in \mathbb{F}_{q^{2}}$, is a common point of $\ell$ and $\Delta$ if and only if $y^{q}+y+(x+t)^{(q+1) / 2}=0$. By (3.2) this occurs when $(x+t)^{(q+1) / 2}=x^{(q+1) / 2}$. For $x=0$, this implies $t=0$. Hence, in this case, $P$ is the only common point of $\ell$ and $\Delta$. In particular, $P \in \Delta_{1}$. For $x \neq 0$, we obtain $(1+t / x)^{(q+1) / 2}=1$. Since all the $\frac{1}{2}(q+1)$-st roots of unity are contained in $\mathbb{F}_{q^{2}}$ and they are pairwise distinct, $\ell$ contains exactly $\frac{1}{2}(q+1)$ points from $\Delta$. The common point of $\ell$ and $\pi$ is the point $\left(1,0, y, y^{2}\right)$ which lies on $\mathscr{C}$, but does not belong to $\Delta_{1}$. Let now $\alpha$ be the plane through $U$ with equation $u_{0} X_{0}+u_{2} X_{2}+u_{3} X_{3}=0$; a point $P\left(1, x, y, y^{2}\right)$ of $\Delta$ lies in $\alpha$ if and only if $u_{0}+u_{2} y+u_{3} y^{2}=0$. Since for every $y \in \mathbb{F}_{q^{2}}$, Equation (3.2) has exactly $\frac{1}{2}(q+1)$ solutions in $x \in \mathbb{F}_{q^{2}}$, statement ii) follows. To prove iii), consider a plane $\beta$ which meets any line through $U$ in exactly one point. By statement i), there are at most $q^{2}+1$ lines through $U$ containing a point of $\Delta$. Hence, $q^{2}+1$ is an upper bound for the number of points in common between $\beta$ and $\Delta$. This proves statement iii).

We need some more notation. For $q \equiv 1(\bmod 4)$, denote by $\Delta^{\prime}$ the set of all points in $\mathscr{H}\left(2, q^{2}\right) \backslash \Delta_{1}$ which are covered by chords of $\mathscr{C}_{0}$. For $q \equiv 3(\bmod 4), \Delta^{\prime}$ will denote the set of all points in $\mathscr{H}\left(2, q^{2}\right)$ which are covered by external lines to $\mathscr{C}_{0}$ in $\pi_{0}$. Clearly, $\Delta^{\prime}$ has size $\frac{1}{2} q(q+1)(q-1)$. Several properties of $\Delta \cup \Delta^{\prime}$ can be deduced from Lemma 3.5. However, we just state one which will be used in Section 5. 
Lemma 3.6. With the notation above,

i) The plane $X_{1}=0$ meets $\Delta \cup \Delta^{\prime}$ in $\frac{1}{2}\left(q^{3}+q+2\right)$ points; any other plane of $\mathrm{PG}\left(3, q^{2}\right)$ has at most $q^{2}+q+2$ points in common with $\Delta \cup \Delta^{\prime}$;

ii) A line through $U$ meets $\Delta U \Delta^{\prime}$ in either $\frac{1}{2}(q+1)$ or 1 or 0 points. More precisely, there are exactly $q^{2}-q$ lines through $U$ sharing $\frac{1}{2}(q+1)$ points with $\Delta \cup \Delta^{\prime}$, and $\frac{1}{2}\left(q^{3}+q+2\right)$ having just one point in $\Delta \cup \Delta^{\prime}$. The former lines meet $\pi$ in the points of the conic $\mathscr{C}$ which are not in $\Delta_{1}$; the latter meet $\pi$ in the points of $\Delta_{1} \cup \Delta^{\prime}$.

The main result of this paper is the following.

Theorem 3.7. The set $\Delta \cup \Delta^{\prime}$ is an ovoid of $\mathscr{H}\left(3, q^{2}\right)$ which cannot be obtained from a Hermitian curve by means of multiple derivation.

The proof of Theorem 3.7 is postponed till Section 5. Meanwhile, we state and prove some properties of the collineation group of $\Delta U \Delta^{\prime}$ which will play a role in its proof.

\section{The subgroup of PGU(4, $\left.q^{2}\right)$ preserving $\Delta \cup \Delta^{\prime}$}

The linear collineation group of $\operatorname{PG}\left(3, q^{2}\right)$ preserving $\mathscr{H}\left(3, q^{2}\right)$ is $\operatorname{PGU}\left(4, q^{2}\right)$. First, we determine the subgroup of $\operatorname{PGU}\left(4, q^{2}\right)$ which preserves $\Delta$. In doing so, we shall be dealing with several collineations from $\operatorname{PGU}\left(4, q^{2}\right)$.

For any $a \in \mathbb{F}_{q^{2}}$, with $a^{q}+a=0$, and for any square $\mu$ in $\mathbb{F}_{q^{2}}$, let

$$
T_{a}:=\left(\begin{array}{cccc}
1 & 0 & 0 & 0 \\
0 & 1 & 0 & 0 \\
a & 0 & 1 & 0 \\
a^{2} & 0 & 2 a & 1
\end{array}\right) ; \quad M_{\mu}:=\left(\begin{array}{cccc}
1 & 0 & 0 & 0 \\
0 & \mu & 0 & 0 \\
0 & 0 & \mu^{(q+1) / 2} & 0 \\
0 & 0 & 0 & \mu^{(q+1)}
\end{array}\right) .
$$

Denote by $\left[T_{a}\right]$ and $\left[M_{\mu}\right]$ the linear collineations associated with the matrices $T_{a}$ and $M_{\mu}$, respectively.

It is easily verified that $\mathbf{T}=\left\{\left[T_{a}\right] \mid a \in \mathbb{F}_{q^{2}}\right\}$ is an elementary Abelian group of order $q$, while $\mathbf{M}=\left\{\left[M_{\mu}\right] \mid \mu \in \mathbb{F}_{q^{2}}^{*}\right\}$ is a cyclic group of order $\frac{1}{2}\left(q^{2}-1\right)$. Furthermore, the group generated by $\mathbf{T}$ and $\mathbf{M}$ is the semidirect product $\mathbf{T} \rtimes \mathbf{M}$.

For any non-zero square $\lambda$ in $\mathbb{F}_{q}^{*}$, let

$$
L_{\lambda}:=\left(\begin{array}{cccc}
1 & 0 & 0 & 0 \\
0 & \lambda & 0 & 0 \\
0 & 0 & 1 & 0 \\
0 & 0 & 0 & 1
\end{array}\right)
$$

Again, $\left[L_{\lambda}\right]$ is the linear collineation associated to the matrix $L_{\lambda}$. Clearly, $\mathbf{L}=$ $\left\{\left[L_{\lambda}\right] \mid \lambda \in \mathbb{F}_{q}^{*}\right\}$ is a cyclic group of order $(q+1) / 2$. Finally, let 


$$
N:=\left(\begin{array}{cccc}
0 & 0 & 0 & 1 \\
0 & 1 & 0 & 0 \\
0 & 0 & 1 & 0 \\
1 & 0 & 0 & 0
\end{array}\right),
$$

and $[N]$ be the associated linear collineation; the collineation group $\mathbf{N}$ generated by $[N]$ has order 2 .

Lemma 4.1. Let $\Gamma$ be the the group generated by all of the above linear collineations. Then

i) $\Gamma$ preserves both $\mathscr{H}\left(3, q^{2}\right)$ and $\Delta$;

ii) $\Gamma$ has two orbits on $\Delta$. One is $\Delta_{1}$ and the other, say $\Delta_{2}$, has size $\frac{1}{2} q(q-1)(q+1)$;

iii) $\Gamma$ acts on $\Delta_{1}$ as a sharply 3-transitive permutation group;

iv) The subgroup $\Phi$ of $\Gamma$ fixing $\Delta_{1}$ pointwise is a cyclic group of order $\frac{1}{2}(q+1)$ and $\Gamma / \Phi \cong \operatorname{PGL}(2, q)$;

v) $\Gamma$ has order $\frac{1}{2} q(q-1)(q+1)^{2}$.

Proof. A straightforward computation shows that each of the above linear collineations preserves both $\mathscr{H}\left(3, q^{2}\right)$ and $\Delta$. This proves the first assertion. Next, take any square $x \in \mathbb{F}_{q^{2}}$. Following Lemma 3.4 , let $\Delta(x)$ be the set of the $q$ points $P_{y}=$ $\left(1, x, y, y^{2}\right)$, satisfying $y^{q}+y=x^{(q+1) / 2}, y \in \mathbb{F}_{q^{2}}$. Then $\Delta_{1}=\Delta(0) \cup P_{\infty}(0,0,0,1)$. Further, let $\Delta_{2}=\bigcup \Delta(x)$, where the union is over the set of non-zero squares of $\mathbb{F}_{q^{2}}$. Then $\left|\Delta_{2}\right|=\frac{1}{2} q\left(q^{2}-1\right)$ and $\Delta=\Delta_{1} \cup \Delta_{2}$. To prove that $\Delta_{2}$ is a full orbit under $\Gamma$, take any two points in $\Delta_{2}$, say $P=\left(1, x, y, y^{2}\right)$ and $Q=\left(1, x^{\prime}, y^{\prime}, y^{\prime 2}\right)$. Since both $x$ and $x^{\prime}$ are non-zero squares in $\mathbb{F}_{q^{2}}$, their ratio $\mu=x / x^{\prime}$ is also a non-zero square element of $\mathbb{F}_{q^{2}}$. The collineation $\left[M_{\mu}\right]$ maps $Q$ onto a point $R=\left(1, x, \bar{y}, \bar{y}^{2}\right) \in \Delta_{2}$. For $a=y-\bar{y}$, the collineation $\left[T_{a}\right]$ takes $R$ onto $P$. This proves the assertion. We now show that $\Gamma$ induces on $\Delta_{1}$ a 3 -transitive permutation group. This depends on the following remarks: the group $\mathbf{T}$ fixes $P_{\infty}$ and acts transitively on the remaining $q$ points in $\Delta_{1}$, whereas $\mathbf{M}$ fixes both $P_{0}$ and $P_{\infty}$ and acts transitively on the remaining $q-1$ points in $\Delta_{1}$. Hence, $\mathbf{T} \rtimes \mathbf{M}$ acts on $\Delta_{1} \backslash\left\{P_{\infty}\right\}$ as a sharply 2-transitive permutation group whose one-point stabiliser is cyclic. Furthermore, $[N]$ interchanges $P_{0}$ and $P_{\infty}$. Following the notation of Lemma 3.4, let $\Phi$ be the normal subgroup of $\Gamma$ which fixes $\pi$ pointwise. Any collineation of $\Phi$ is associated with a diagonal matrix of type

$$
\left(\begin{array}{llll}
1 & 0 & 0 & 0 \\
0 & \rho & 0 & 0 \\
0 & 0 & 1 & 0 \\
0 & 0 & 0 & 1
\end{array}\right),
$$

with $\rho \in \mathbb{F}_{q^{2}}^{*}$; such collineation preserves $\Delta$ if and only if $\rho^{(q+1) / 2}=1$. This shows that $\Phi=\mathbf{L}$. Hence, $\Phi$ is a cyclic group of order $(q+1) / 2$. Let $G=\Gamma / \Phi$ be the linear 
collineation group induced by $\Gamma$ on $\pi$. Then $G$ is the linear collineation group of $\pi$ which preserves $\Delta_{1}$. Actually, $G$ also preserves the Baer subplane $\pi_{0}$ as defined in III) of Lemma 3.4, since the associated Baer involution $\beta$ centralises $G$. By IV) of Lemma $3.4, G$ is a linear collineation group of $\pi_{0}$ which acts 3-transitively on a conic $\mathscr{C}_{0}$ of $\pi_{0}$. Thus, $G \cong \operatorname{PGL}(2, q)$ acts on $\mathscr{C}_{0}$ as $\operatorname{PGL}(2, q)$ in its unique sharply 3 -transitive permutation representation. In particular, $G$ has order $q(q-1)(q+1)$, and hence v) holds.

In the previous proof, we have also shown that $\Gamma$ coincides with the subgroup of $\operatorname{PGU}\left(4, q^{2}\right)$ which preserves both $\Delta_{1}$ and $\Delta_{2}$. Actually, this result can be improved with little more effort.

Lemma 4.2. The group $\Gamma$ is the subgroup of $\mathrm{PGU}\left(4, q^{2}\right)$ which preserves $\Delta$.

Proof. Assume, to the contrary, that the subgroup of $\operatorname{PGU}\left(4, q^{2}\right)$ which preserves $\Delta$ acts transitively on $\Delta$. Then the size of $\Delta$ should divide the order of $\operatorname{PGU}\left(3, q^{2}\right)$, that is, $\frac{1}{2}(q+1)\left(q^{2}-q+2\right)$ should divide $q^{6}(q+1)^{3}(q-1)^{2}\left(q^{2}-q+1\right)$. Let $d$ be a prime divisor of $q^{2}-q+2$. Thus $d$ divides $(q-1)^{2}(q+1)^{3}$ too. This is possible only for $d=2$. Hence, $q^{2}+q-2=2^{m}$ for an integer $m \geqslant 1$. We show that this cannot occur for $q \geqslant 5$. First, assume that $m=2 n$ is even and write $q^{2}-q+2=2^{2 n}$ in the equivalent form $\left(2^{n+1}+(2 q-1)\right)\left(2^{n+1}-(2 q-1)\right)=7$, whence $2^{n+1}+2 q-1=7$ and $2^{n+1}-(2 q-1)=1$. This only occurs for $q=2, n=1$. For the case $m=2 n+1$, write $q^{2}-q+2=2^{2 n+1}$ as $q(q-1)=2\left(2^{n}+1\right)\left(2^{n}-1\right)$. This yields $k q=2^{n} \pm 1$ and $\frac{1}{2 k}(q-1)=2^{n} \mp 1$ for a divisor $k$ of $q-1$. Then $k q-\frac{1}{2 k}(q-1)=2$, which is only possible for $q=3, n=1$ and $k=1$, since $k q-\frac{1}{2 k}(q-1)>(q-1)\left(k-\frac{1}{k}\right)>$ $\frac{1}{2} k(q-1)$.

We now turn our attention to $\Delta^{\prime}$.

Lemma 4.3. The group $\Gamma$ preserves $\Delta^{\prime}$. More precisely, $\Delta^{\prime}$ is an orbit under $\Gamma$.

Proof. Using the notation of Lemma 3.4, $\Gamma$ preserves the plane $\pi$ and induces on $\pi$ a linear collineation group $G \cong \operatorname{PGL}(2, q)$ that leaves both $\mathscr{C}_{0}$ and $\mathscr{H}\left(2, q^{2}\right)$ invariant. In particular, $\Gamma$ preserves the set of all chords of $\mathscr{C}_{0}$, as well as that of external lines to $\mathscr{C}_{0}$. Hence, it leaves $\Delta^{\prime}$ invariant. To prove that $G$ is transitive on $\Delta^{\prime}$, it is enough to show that the stabiliser $G_{P}$ of a point $P \in H\left(2, q^{2}\right) \backslash \Delta_{1}$ has order 2 . As $P \notin \pi_{0}$, there is only one line of $\pi_{0}$ through $P$, say $\ell$. Since tangents to $\mathscr{C}_{0}$ are also tangents to $\mathscr{H}\left(2, q^{2}\right), \ell$ is either a chord of $\mathscr{C}_{0}$ or an external line to $\mathscr{C}_{0}$. Thus, the stabiliser $G_{\ell}$ of $\ell$ is a dihedral group $D_{q \pm 1}$ of order $2(q \pm 1)$, where + or - occurs depending on whether $\ell$ is an external line or a chord. The central involution of $D_{q \pm 1}$ fixes $\ell$ pointwise, whereas each of the $q \pm 1$ non-central involutions of $D_{q \pm 1}$ has exactly two fixed points, both in $\pi_{0}$, hence distinct from $P$. Choose now any element $g \in D_{q \pm 1}$ of order greater than 2. To complete the proof we have to show that $g(P) \neq P$. If $\ell$ is an external line to $\mathscr{C}_{0}$, then $g$ has no fixed point on $\ell$; when $\ell$ is a chord, $g$ fixes the common points of $\ell$ and $\mathscr{C}_{0}$ but no other point on $\ell$. 
Our final result is the following theorem.

Theorem 4.4. The group $\Gamma$ is the subgroup of $\operatorname{PGU}\left(4, q^{2}\right)$ which preserves $\Delta \cup \Delta^{\prime}$.

Proof. By virtue of the last two Lemmas, we have only to prove that any collineation $g \in \mathrm{PGU}\left(3, q^{2}\right)$ preserving $\Delta U \Delta^{\prime}$ must also preserve $\Delta$. By i) of Lemma 3.6, $g$ preserves the plane $\pi$ with equation $X_{1}=0$. Since $U=(0,1,0,0)$ is the pole of $\pi$ with respect to the unitary polarity associated with $\mathscr{H}\left(3, q^{2}\right)$, it turns out that $g$ fixes $U$. By ii) of Lemma 3.6, $g$ preserves the conic $\mathscr{C}$ of $\pi$. Since $g$ preserves $\mathscr{H}\left(2, q^{2}\right)=$ $\mathscr{H}\left(3, q^{2}\right) \cap \pi$ and $\Delta_{1}=\mathscr{H}\left(2, q^{2}\right) \cap \mathscr{C}=\mathscr{C}_{0}$, it follows that $g$ preserves both $\Delta_{1}$ and $\mathscr{C} \backslash \Delta_{1}$. Again, by ii) of Lemma 3.6, the latter assertion yields that $g$ preserves not only $\Delta_{1}$ but also $\Delta \backslash \Delta_{1}$. This can only happen if $g$ preserves $\Delta$.

\section{The proof of Theorem 3.7}

We keep our previous notation. We first prove that $\mathcal{O}=\Delta U \Delta^{\prime}$ is an ovoid. Since $\mathcal{O}$ has the right size, $q^{3}+1$, it is enough to show that no two distinct points in $\mathcal{O}$ are conjugate under the unitary polarity associated with $\mathscr{H}\left(3, q^{2}\right)$. As $\Delta_{1} \cup \Delta_{2}$ lies in the plane $\pi$, which is not tangent to $\mathscr{H}\left(3, q^{2}\right)$, our assertion is true for any two distinct points in $\Delta_{1} \cup \Delta^{\prime}$. It remains to prove that no point $P \in \Delta_{2}=\Delta \backslash \Delta_{1}$ is conjugate to another point in $\Delta \cup \Delta^{\prime}$. Since, by ii) of Lemma $4.1, \Gamma$ acts transitively on $\Delta_{2}$, we may assume $P\left(1,1,-\frac{1}{2}, \frac{1}{4}\right)$. The plane $\alpha_{P}$, tangent to $\mathscr{H}\left(3, q^{2}\right)$ at $P$, has equation $X_{0}-4 X_{1}-4 X_{2}+4 X_{3}=0$. We have to verify that both of the following statements hold:

i) $\alpha_{P}$ has no points in $\Delta$ except $P$;

ii) $\alpha_{P}$ meets $\pi$ in a line disjoint from $\Delta_{1} \cup \Delta^{\prime}$.

Let $Q=\left(1, x, y, y^{2}\right) \in \Delta_{2}$ be a point of $\alpha_{P}$. Then by Lemma 3.2, $x=\xi^{2}$ with $\xi \in \mathbb{F}_{q^{2}}$. In this case, both $1-4 \xi^{2}-4 y+4 y^{2}=0$ and $y^{q}+y+\xi^{q+1}=0$. The former equation gives $y= \pm \frac{1}{2}(2 \xi+1)$; it follows that $\left( \pm \xi^{q}-1\right)( \pm \xi-1)=0$. This yields $\xi= \pm 1$. Thus, $x=1$ and either $y=-\frac{1}{2}$, or $y=\frac{3}{2}$. As $q$ is odd, the latter condition is impossible. Hence, $Q$ is the only common point of $\alpha$ and $\Delta_{2}$.

To verify ii), we consider the line $\ell=\alpha_{P} \cap \pi$ with equation $X_{0}-4 X_{2}+4 X_{3}=0$, and we show that $\ell$ is disjoint from $\Delta^{\prime}$.

We first deal with the case $q \equiv 1(\bmod 4)$. For any chord $r$ of $\mathscr{C}_{0}$, compute the coordinates of the point $R=\ell \cap r$. Let $R_{1}=\left(1, u, u^{2}\right)$ and $R_{2}=\left(1, v, v^{2}\right)$, with $u^{q}+u=0$, $v^{q}+v=0$, be the common points of $r$ and $\mathscr{C}_{0}$. Since $r$ has equation $u v X_{0}-(u+v) X_{2}+$ $X_{3}=0$, we have $R=(4(u+v-1), 4 u v-1,4 u v-u-v)$. Let

$$
f=4(u+v-1)^{q}(4 u v-u-v)+4(u+v-1)(4 u v-u-v)^{q}+2(4 u v-1)^{q+1} .
$$

Then $f=0$ if and only if $R \in \mathscr{H}\left(2, q^{2}\right)$. By a straightforward computation,

$$
\begin{aligned}
f= & 4(u+v-1)^{q}(u+v-4 u v)+4(u+v-1)(u+v-4 u v)^{q} \\
& +2(4 u v-1)^{q+1}=4\left(1+4 v^{2}\right) u^{2}-16 v u+4 v^{2}+1 .
\end{aligned}
$$


This shows that $f=0$ implies that

$$
u=\frac{4 v+\left(4 v^{2}-1\right) j}{2\left(1+4 v^{2}\right)}, \quad j^{2}=-1 .
$$

As $q \equiv 1(\bmod 4)$, we have $j^{q}=j$. Taking $u^{q}+u=0, v^{q}+v=0$ into account, we see that $f=0$ yields

$$
0=u^{q}+u=\frac{4 v-1}{2(1+4 v)}\left(j+j^{q}\right) .
$$

Therefore, $q \equiv 1(\bmod 4)$ implies $f \neq 0$ and ii) follows for this case.

If $q \equiv 3(\bmod 4)$, we have to consider an external line $r$ to $\mathscr{C}_{0}$. Since $r$ meets $\mathscr{C}$ in two distinct points, $r$ can be regarded as the line joining the point $R_{1}\left(1, u, u^{2}\right)$, with $u^{q}+u \neq 0$, and its image $R_{2}\left(1,-u^{q}, u^{2 q}\right)$ under the Baer involution associated with $\pi_{0}$, see statement III) of Lemma 3.4. Hence, $r$ has equation $X_{3}+\left(u^{q}-u\right) X_{2}-$ $u^{q+1} X_{0}=0$. The common point of $r$ and $\ell$ is $R=\left(4\left(u^{q}-u+1\right), 4 u^{q+1}+1,4 u^{q+1}-\right.$ $\left.u^{q}+u\right)$. Let

$$
\begin{aligned}
f= & 4\left(u^{q}-u+1\right)^{q}\left(4 u^{q+1}-u^{q}+u\right) \\
& +4\left(u^{q}-u+1\right)\left(4 u^{q+1}-u^{q}+u\right)^{q}+2\left(4 u^{q+1}+1\right)^{q+1} .
\end{aligned}
$$

Then $R \in \mathscr{H}\left(2, q^{2}\right)$ if and only if $f=0$. By a direct computation $f=2\left[4\left(u^{q}+u\right)^{2}+\right.$ $\left.\left(4 u^{q+1}+1\right)^{2}\right]$. Therefore, $f=0$ implies that $2\left(u^{q}+u\right)=j\left(4 u^{q+1}+1\right)$ with $j^{2}=-1$, whence $4 u^{q+1}+1 \neq 0$ and

$$
j=2 \frac{u^{q}+u}{4 u^{q+1}+1}
$$

This yields $j \in \mathbb{F}_{q}$, contradicting $q \equiv 3(\bmod 4)$, and completes the proof of ii).

Finally, assume by way of contradiction that $\mathcal{O}$ is obtained by a multiple derivation. According to Lemma 2.2, there is a homology group $\Psi$ of order $q+1$ preserving $\mathcal{O}$. Let $\alpha$ be its axis; the pole $A$ of $\alpha$ is the centre of the elements of $\Psi$. By Theorem $4.4, \Psi$ is a subgroup of $\Gamma$; hence, it preserves $\pi$. However, $\Psi$ is not a subgroup of $\Phi$, since, by iv) of Lemma 4.1, the subgroup $\Phi$ of $\Gamma$ fixing $\pi$ pointwise has order $\frac{1}{2}(q+1)$. In particular, $\alpha \neq \pi$. Hence, $\Psi$ acts faithfully on $\pi$. In other words, the linear collineation group $H$ induced by $\Psi$ on $\pi$ has order $q+1$. Actually, $H$ is a homology group of $\pi$ whose axis is the common line of $\alpha$ and $\pi$ and whose centre is the point of intersection of $\pi$ and the line joining $A$ and $U$. By ii) of Lemma 3.5, $H$ preserves the conic $\mathscr{C}$ of $\pi$. This leads to a contradiction, as no homology of order $t>2$ preserves a conic.

\section{References}

[1] R. D. Baker, G. L. Ebert, G. Korchmáros, T. Szőnyi, Orthogonally divergent spreads of Hermitian curves. In: Finite geometry and combinatorics (Deinze, 1992), volume 191 of London Math. Soc. Lecture Note Ser., 17-30, Cambridge Univ. Press 1993.

MR 94k:51013 Zbl 0804.51013 
[2] A. Barlotti, Un'estensione del teorema di Segre-Kustaanheimo. Boll. Un. Mat. Ital. (3) 10 (1955), 498-506. MR 17,776b Zbl 0066.38901

[3] A. E. Brouwer, H. A. Wilbrink, Ovoids and fans in the generalized quadrangle $Q(4,2)$. Geom. Dedicata 36 (1990), 121-124. MR 91h:51007 Zbl 0716.51007

[4] A. Cossidente, G. Korchmáros, Transitive ovoids of the Hermitian surface of $\operatorname{PG}\left(3, q^{2}\right)$, with $q$ even. J. Combin. Theory Ser. A. 101 (2003), 117-130.

[5] A. Gunawardena, Primitive ovoids in $O_{8}^{+}(q)$. J. Combin. Theory Ser. $A 89$ (2000), 70-76. MR 2000i:51025 Zbl 0961.51007

[6] J. W. P. Hirschfeld, Finite projective spaces of three dimensions. Oxford Univ. Press 1985. MR 87j:51013 Zbl 0574.51001

[7] J. W. P. Hirschfeld, Projective geometries over finite fields. Oxford Univ. Press 1998. MR 99b:51006 Zbl 0899.51002

[8] W. M. Kantor, R. A. Liebler, The rank 3 permutation representations of the finite classical groups. Trans. Amer. Math. Soc. 271 (1982), 1-71. MR 84h:20040 Zbl 0514.20033

[9] P. B. Kleidman, The 2-transitive ovoids. J. Algebra 117 (1988), 117-135. MR 89j:51008 Zbl 0652.51013

[10] G. Panella, Caratterizzazione delle quadriche di uno spazio (tridimensionale) lineare sopra un corpo finito. Boll. Un. Mat. Ital. (3) 10 (1955), 507-513. MR 17,776c Zbl 0066.38902

[11] B. Segre, Ovals in a finite projective plane. Canad. J. Math. 7 (1955), 414-416. MR 17,72g Zbl 0065.13402

[12] B. Segre, Forme e geometrie hermitiane, con particolare riguardo al caso finito. Ann. Mat. Pura Appl. (4) 70 (1965), 1-201. MR 35 \#4802 Zbl 0146.16703

[13] J. A. Thas, Ovoids and spreads of finite classical polar spaces. Geom. Dedicata 10 (1981), 135-143. MR 82g:05031 Zbl 0458.51010

[14] J. A. Thas, Old and new results on spreads and ovoids of finite classical polar spaces. In: Combinatorics '90 (Gaeta, 1990), volume 52 of Ann. Discrete Math., 529-544, NorthHolland 1992. MR 93h:51005 Zbl 0767.51004

[15] J. A. Thas, Ovoids, spreads and $m$-systems of finite classical polar spaces. In: Surveys in combinatorics, 2001 (Sussex), volume 288 of London Math. Soc. Lecture Note Ser., 241-267, Cambridge Univ. Press 2001. MR 2003a:51003 Zbl 0986.51005

[16] J. A. Thas, S. E. Payne, Spreads and ovoids in finite generalized quadrangles. Geom. Dedicata 52 (1994), 227-253. MR 95m:51005 Zbl 0804.51007

Received 30 December, 2002

L. Giuzzi, Dipartimento di Matematica, Università degli Studi di Brescia, via Valotti 9, 25133 Brescia, Italy

Email: giuzzi@ing.unibs.it, giuzzi@dmf.unicatt.it

G. Korchmáros, Dipartimento di Matematica, Università degli Studi della Basilicata, Contrada Macchia Romana, 85100 Potenza, Italy

Email: korchmaros@unibas.it 